Arch. math. Logik 25 (1985), 91-98

\title{
APPLICATION OF A TAUBERIAN THEOREM TO FINITE MODEL THEORY*
}

Kevin J. Compton

\begin{abstract}
An extension of a Tauberian theorem of Hardy and Littlewood is proved. It is used to show that, for classes of finite models satisfying certain combinatorial and growth properties, Cesàro probabilities (limits of average probabilities over second order sentences) exist. Examples of such classes include the class of unary functions and the class of partial unary functions. It is conjectured that the result holds for the usual notion of asymptotic probability as well as Cesàro probability. Evidence in support of the conjecture is presented.
\end{abstract}

\section{Introduction}

Suppose that $a(x)=\sum_{n=0}^{\infty} a_{n} x^{n}, b(x)=\sum_{n=0}^{\infty} b_{n} x^{n}, a(x)$ has radius of convergence $R>0$, $\lim _{x \rightarrow R} a(x)=\infty$, and $\lim _{n \rightarrow \infty} b_{n} / a_{n}=L$. It is easy to show that

$$
\lim _{x \rightarrow R} \frac{b(x)}{a(x)}=L
$$

(see Theorem 57 of Hardy [8] or Section 7.5 of Titchmarsh [12]). This is an Abelian theorem - "... roughly, one which asserts that if a sequence or function behaves regularly, then some average of the sequence or function behaves regularly" ([8, p. 148]). The sequence $b_{n}$ behaves regularly with respect to $a_{n}$, from which it follows that the average defined by (1) behaves regularly. Theorem 1 , the main theorem of the paper, is a partial converse to this theorem. Obtaining a converse requires imposition of the conditions $a_{n} \sim n^{\alpha}, \alpha>-1, b_{n}=O\left(a_{n}\right)$ on hypothesis (1); from this it follows the somewhat weakened conclusion

$$
\lim _{n \rightarrow \infty} \frac{1}{n} \sum_{k=0}^{n-1} \frac{b_{k}}{a_{k}}=L
$$

* Eingegangen am 17. 7. 1984.

1980 Mathematics Subject Classification: $40 \mathrm{G} 05,40 \mathrm{G} 10,03 \mathrm{C} 13$.

Key Words and Phrases: Tauberian theorem, finite model theory, asymptotic probability, Cesàro probability, unary function. 
If, further, $b_{n} / a_{n}$ is a slowly decreasing sequence (see Section 1 for definitions) then $\lim _{n \rightarrow \infty} b_{n} / a_{n}=L$. These are Tauberian theorems - “...corrected forms of false converses of Abelian theorems" ([8, p. 149]).

Theorem 1 restricted to the case $a_{n}=1$ is a Tauberian theorem of Hardy and Littlewood (Theorems 92 and 106 of Hardy [8]). The proof of Theorem 1 presented here is similar to Rubel's proof of the Hardy-Littlewood theorem using Littlewood and Pólya means [11].

The significance of Theorem 1 lies in an application to finite model theory. Fagin [5] asked whether first order sentences about unary functions have asymptotic probabilities. Lynch [10] answered the question affirmatively, even for structures consisting of several unary and partial unary functions. Does Lynch's result extend to monadic second order sentences? This is a natural question because many combinatorial properties not expressible in first order logic are expressible in monadic second order logic. There is some hope of success because a result in Compton [3] shows that under certain general conditions extended asymptotic probabilities - quantities related to expression (1) - will exist. Theorem 1 then applies by supposing a growth condition - one broad enough to include the class of unary functions - on the class of structures considered. The conclusion, specified in Theorem 2, is that Cesàro probabilities - quantities related to expression (2) - exist for monadic second order sentences. Strictly speaking this does not extend Lynch's result because Cesàro probabilities may exist when asymptotic probabilities do not: unfortunately, Lynch's techniques do not carry over in an obvious way for monadic second order sentences so at present Theorem 2 is the best result in this direction.

It is worthwhile to compare Lynch's approach in [10] to the one in [3] and here. In both approaches the logical arguments rely on Ehrenfeucht games. Lynch's use of these games is closely tied to the structure of unary functions while in [3] it is more general, depending only on broad combinatorial properties. Lynch, consequently, obtains an algorithm for computing the probabilities of first order sentences while the methods of [3] produce no such algorithm (but in some cases a closer analysis will yield an algorithm; see [4]). Lynch's main combinatorial tool is a generalization of the inclusion-exclusion principle, while in [3] it is enumeration by means of generating series. These series must have positive radius of convergence, hence will not work for classes of more than one unary or partial unary function, in contrast to Lynch's techniques. Finally, the main analytic tools for demonstrating convergence of asymptotic probabilities are, for Lynch, a theorem similar to the Monotone and Dominated Convergence Theorems of real analysis, and here, the Tauberian theorem which is the subject of the paper.

The paper is organized as follows. Section 1 contains preliminary definitions and results. Section 2 contains the main result and application. Section 3 presents a conjecture which, if true, subsumes part of Lynch's results and part of the results in Section 2. Evidence for the conjecture is presented.

My first proof of Theorem 1 was somewhat like the proof of the Hardy-Littlewood Tauberian Theorem from Wiener's Tauberian Theorem (see Hardy [8, p. 300]). I 
would like to thank Lee Rubel for suggesting I find a Wiener-free alternative and directing my attention to [11]. I would also like to thank the faculty and staff of Mathematics Department of the University of Illinois at Champaign-Urbana for extending their hospitality and services during my visit Spring semester, 1984.

\section{Preliminaries}

An often occurring Tauberian condition is that of a sequence being slowly decreasing. The sequence $a_{n}, n \geqq 0$, is slowly decreasing if for every $\varepsilon>0$ there are $N \geqq 0$ and $\xi$ between 0 and 1 such that $a_{n}-a_{m}>-\varepsilon$ when $n \geqq N$ and $\xi n \leqq m \leqq n$ (the terminology is misleading: slowly decreasing does not imply monotone).

For notation and fundamental concepts from model theory consult Chang and Keisler [1].

Fix a finite relational language $L$ and let $\mathscr{C}$ be a class of $L$-structures closed under isomorphism. Let $\mathscr{A}_{n}$ be the set of structures in $\mathscr{C}$ with universe $n=\{0,1, \ldots, n-1\}$ and $a_{n}=\left|\mathscr{A}_{n}\right|$. The exponential generating series of $\mathscr{C}$ is

$$
\sum_{n=0}^{\infty} \frac{a_{n}}{n !} x^{n}
$$

(assume $a_{0}=1$ ). For a sentence $\varphi$ let $\mu_{n}^{\mathscr{E}}(\varphi)$ be the fraction of structures in $\mathscr{A}_{n}$ that satisfy $\varphi$. The labeled asymptotic probability of $\varphi$ is

$$
\mu^{\mathscr{E}}(\varphi)=\lim _{n \rightarrow \infty} \mu_{n}^{\mathscr{E}}(\varphi)
$$

whenever this limit exists and otherwise undefined. The superscript $\mathscr{C}$ will be deleted when the class $\mathscr{C}$ is clear from context. The labeled Cesàro probability of $\varphi$ is

$$
\mu^{*}(\varphi)=\lim _{n \rightarrow \infty} \frac{1}{n} \sum_{k=0}^{n-1} \mu_{k}(\varphi)
$$

whenever this limit exists and otherwise undefined.

Let $\mathfrak{U}$ be an $L$-structure. Define a binary relation $\sim$ on the universe of $\mathfrak{U}$ as follows. $a \sim b$ if for some relation symbol $R$ in $L$ and sequences $\bar{x}, \bar{y}, \bar{z}$ of variables

$$
\mathfrak{Q}=(\exists \bar{x}, \bar{y}, \bar{z}) R(\bar{x}, a, \bar{y}, b, \bar{z}) .
$$

Let $\sim$ * be the least equivalence relation extending $\sim$. The *-equivalence classes are called components of $\mathfrak{U}$. Also, a substructure $\mathfrak{B}$ of $\mathfrak{U}$ is called a component of $\mathfrak{U}$ if its universe is a component of $\mathfrak{A}$. A class $\mathscr{C}$ is closed under components if whenever $\mathfrak{U} \in \mathscr{C}$ and $\mathfrak{B}$ is a component of $\mathfrak{U}$ then $\mathfrak{B} \in \mathscr{C}$.

Since $L$ is relational, unary functions will be defined as relations. Suppose $L$ consists of a single symbol $R$, a binary relation symbol. The class of partial unary functions is the set of all $L$-structures $\mathfrak{A}$ such that

$$
\mathfrak{U} \models(\forall x, y, z)[R(x, y) \wedge R(x, z) \rightarrow y=z] .
$$

The class of unary functions satisfy the further condition

$$
\mathfrak{U}=\forall x \exists y R(x, y) .
$$


In the sequel it will be important to note that both these classes are closed under disjoint unions and components.

\section{The Main Theorem and Application}

Theorem 1. Let $a_{n} \sim n^{\alpha}, \alpha>-1, a_{n} \neq 0$ for $n \geqq 0, b_{n}=O\left(a_{n}\right)$,

$$
a(x)=\sum_{n=0}^{\infty} a_{n} x^{n}, \quad b(x)=\sum_{n=0}^{\infty} b_{n} x^{n} .
$$

Then the following are equivalent.

$$
\begin{aligned}
& \lim _{x \rightarrow 1-} b(x) / a(x)=L . \\
& \lim _{n \rightarrow \infty} \frac{1}{n} \sum_{k=0}^{n-1} \frac{b_{k}}{a_{k}}=L .
\end{aligned}
$$

Moreover, if (i) holds and $b_{n} / a_{n}$ is slowly decreasing then $\lim _{n \rightarrow \infty} b_{n} / a_{n}=L$.

Proof. Without loss of generality $b_{0}=0$. Define $f:[0, \infty) \rightarrow \mathbb{R}$ by $f(x)=b_{n} / a_{n}$, $n \leqq x<n+1$. It is easy to verify that

$$
\begin{gathered}
a\left(e^{-x}\right) \sim \int_{0}^{\infty} t^{\alpha} e^{-x t} d t, \\
b\left(e^{-x}\right)=\int_{0}^{\infty} t^{\alpha} e^{-x t} f(t) d t+o\left(a\left(e^{-x}\right)\right)
\end{gathered}
$$

as $x \rightarrow 0+$ (for the latter observe that $a\left(e^{-x}\right) \rightarrow \infty$ as $x \rightarrow 0+$ ), and

(where $n=\lfloor x\rfloor$ ) as $x \rightarrow \infty$.

$$
\frac{1}{n} \sum_{k=0}^{n-1} \frac{b_{k}}{a_{k}} \sim \frac{1}{x} \int_{0}^{x} f(t) d t
$$

The upper and lower Littlewood means of $f(x)$ are

$$
\begin{aligned}
& A(\xi)=\limsup _{x \rightarrow 0+} \int_{0}^{\infty} t^{\xi} e^{-x t} f(t) d t \mid \int_{0}^{\infty} t^{\xi} e^{-x t} d t, \\
& \lambda(\xi)=\liminf _{x \rightarrow 0+}(\text { same }),
\end{aligned}
$$

for $-1<\xi<\infty$. Values for $\xi=-1, \infty$ are defined by continuity. The upper and lower Pólya means of $f(x)$ are

$$
\begin{aligned}
& \Pi(\xi)=\limsup _{x \rightarrow \infty} \frac{1}{x-\xi x} \int_{\xi x}^{x} f(t) d t, \\
& \pi(\xi)=\liminf _{x \rightarrow \infty} \text { (same), }
\end{aligned}
$$

for $0 \leqq \xi<1$. Values at $\xi=1$ are defined by continuity. 
Thus, (i) holds precisely when $A(\alpha)=\lambda(\alpha)=L$ and (ii) holds precisely when $\Pi(0)$ $=\pi(0)=L$. The theorem then follows from the following results of Rubel [11]:

(a) $\Lambda(\xi)=\lambda(\xi)=L$ for some $\xi,-1 \leqq \xi \leqq \infty$, iff $A(\xi)=\lambda(\xi)=L$ for all such $\xi$.

(b) $\Pi(\xi)=\pi(\xi)=L$ for some $\xi, 0 \leqq \xi \leqq 1$, iff $\Pi(\xi)=\pi(\xi)=L$ for all such $\xi$.

(c) $\lambda(\infty)=\Pi(1), \lambda(\infty)=\pi(1)$.

To prove the second part of the theorem note that if $b_{n} / a_{n}$ is slowly decreasing then for a given $\varepsilon>0$ there are $N$ and $\xi, 0<\zeta<1$, such that $f(x)-f(t)>-\varepsilon$ when $x \geqq N$ and $\xi x \leqq t \leqq x$. Thus $f(t)-f(x)>-\varepsilon$ when $x \geqq N, x \leqq t \leqq x / \xi$. Therefore, for $x \geqq N$

$$
\frac{1}{x-\xi x} \int_{\xi x}^{x} f(t) d t-\varepsilon \leqq f(x) \leqq \frac{1}{x / \xi-x} \int_{x}^{x / \xi} f(t) d t+\varepsilon
$$

whence

$$
L-\varepsilon \leqq \liminf _{x \rightarrow \infty} f(x) \leqq \limsup _{x \rightarrow \infty} f(x) \leqq L+\varepsilon
$$

Letting $\varepsilon$ approach 0 it follows that $\lim _{n \rightarrow \infty} b_{n} / a_{n}=L$.

Theorem 2. Let $\mathscr{C}$ be closed under disjoint unions and components with $\left|\mathscr{A}_{n}\right| / n ! \sim A n^{\alpha} R^{-n},\left|\mathscr{A}_{n}\right| \neq 0$ for $n \geqq 0, \alpha>-1, A, R>0$. Then $\mu^{\sharp}(\varphi)$ exists for each monadic second order $\varphi$.

Proof. Let $a_{n}=\left|\mathscr{A}_{n}\right|, b_{n}=\left|\left\{\mathfrak{U} \in \mathscr{A}_{n}: \mathfrak{U} \mid=\varphi\right\}\right|$,

$$
a(x)=\sum_{n=0}^{\infty} \frac{a_{n}}{n !} x^{n}, \quad b(x)=\sum_{n=0}^{\infty} \frac{b_{n}}{n !} x^{n} .
$$

Since $\lim _{x \rightarrow R^{-}} a(x)=\infty$ it follows from Theorem 4.3 of Compton [3] that $\lim _{x \rightarrow R} b(x) / a(x)$ exists; let its value be $L$. Put

$$
\bar{a}(x)=\sum_{n=0}^{\infty} \bar{a}_{n} x^{n}, \quad \bar{b}(x)=\sum_{n=0}^{\infty} \bar{b}_{n} x^{n}
$$

where $\bar{a}_{n}=a_{n} R^{n} /(A n !), \bar{b}_{n}=b_{n} R^{n} /(A n !)$. Hence $\bar{a}_{n} \sim n^{\alpha}$ and $\lim _{x \rightarrow 1-} \bar{b}(x) / \bar{a}(x)=L$. By Theorem $1, \mu^{\sharp}(\varphi)=\lim _{n \rightarrow \infty} \frac{1}{n} \sum_{k=0}^{n-1} \bar{b}_{k} / \bar{a}_{k}=L$.

Corollary 3. If $\mathscr{C}$ is the class of unary functions or the class of partial unary functions then $\mu^{\sharp}(\varphi)$ exists for each monadic second order $\varphi$.

Proof. If $\mathscr{C}$ is the class of unary functions then $\left|\mathscr{A}_{n}\right|=n^{n}$ and $n^{n} / n ! \sim(2 \pi)^{-1 / 2} n^{-1 / 2} e^{n}$ by Stirling's formula. If $\mathscr{C}$ is the class of partial unary functions $\left|\mathscr{A}_{n}\right|=(n+1)^{n}$ and $(n+1)^{n} / n ! \sim e(2 \pi)^{-1 / 2} n^{-1 / 2} e^{n}$. Thus, Theorem 2 applies. 


\section{A Conjecture}

When $\alpha \geqq 0$ Theorem 2 can be strengthened: $\mu^{\sharp}(\varphi)$ may be replaced by $\mu(\varphi)$. This follows from Theorem 6.6 of Compton [3] which says that for $\bar{a}_{n}$ as in the proof of Theorem 2 if there is a fixed bound for $\bar{a}_{n-i} / \vec{a}_{n}$ for all sufficiently large $n$ and all $i \leqq n$ then $\mu(\varphi)$ exists for each monadic second order $\varphi$. Whether Theorem 2 can be similarly strengthened when $-1<\alpha<0$ is open. A possible approach would be to show that every monadic second order sentence can be expressed as a Boolean combination of monadic second order sentences $\varphi$ where $\mu_{n}(\varphi)$ is slowly decreasing.

Conjecture. Theorem 2 remains true when $\mu^{*}(\varphi)$ is replaced by $\mu(\varphi)$.

If this conjecture is true then Lynch's result in the case of a single unary function or a single partial unary function extends to monadic second order sentences. It is unlikely that techniques presented here would apply to the case of more than one function or partial function. Indeed, labeled asymptotic probabilities or even Cesàro probabilities of monadic second order sentences may not always exist in the case of more than one function. Compare the class of all structures for a relational language containing at least one non-unary relation: Glebskii, Kogan, Liogon'kii, and Talanov [6], and independently, Fagin [5], proved for this class that $\mu(\varphi)$ exists for first order $\varphi$, but Kaufmann and Shelah [9] showed that this is not true for monadic second order $\varphi$. Their example shows in fact that there will be monadic second order sentences without limiting probabilities for any reasonable notion of asymptotic probability (including Cesàro probability). This may be a common phenomenon in quickly growing classes.

Theorem 4 lends plausibility to the conjecture. It requires the following definition.

Definition. Let $\mathscr{C}$ be closed under disjoint unions and components. Add unary relation symbols $R_{1}, R_{2}, \ldots, R_{n}$ to the language of this class. If $\mathscr{U} \in \mathscr{C}$, a component $N$-coloring of $\mathfrak{A}$ is an expansion of $\mathfrak{U}$ to the new language such that the interpretations of $R_{1}, R_{2}, \ldots, R_{N}$ partition the universe of $\mathfrak{U}$ and the components of $\mathfrak{U}$ refine this partition. That is, elements within the same component will have the same color $R_{i}$. The component $N$-coloring class $\mathscr{D}$ for $\mathscr{C}$ is the class of all component $N$-colorings of structures in $\mathscr{C} . \mathscr{D}$ is clearly closed under disjoint unions and components but in general $\mu^{\mathscr{C}}(\varphi)$ is not $\mu^{\mathscr{D}}(\varphi)$ (when they exist): structures from $\mathscr{C}$ with many components have greater weight in $\mathscr{D}$ because their components can be colored many ways.

Theorem 4. Let $\mathscr{C}$ be closed under disjoint unions and components with $\left|\mathscr{A}_{n}\right| / n$ ! $\sim A n^{\alpha} R^{-n}, \alpha>-1, A, R>0$. Let $N$ be an integer not less than $1 /(1+\alpha)$ and let $\mathscr{D}$ be the component $N$-coloring class for $\mathscr{C}$. Then $\mu^{\mathscr{D}}(\varphi)$ exists for all monadic second order $\varphi$ in the language of $\mathscr{D}$. 
Proof. Let $\left|\mathcal{\alpha}_{n}\right|=a_{n}$. The exponential generating series for $\mathscr{C}$ is

$$
a(x)=\sum_{n=0}^{\infty} \frac{a_{n}}{n !} x^{n} .
$$

By standard combinatorial methods the exponential generating series for $\mathscr{D}$ is $a(x)^{N}$ (i.e., the formal $N^{\text {th }}$ power of the series; see Compton [2] or Goulden and Jackson [7] for a discussion of these methods). Let

$$
a(x)^{N}=a^{*}(x)=\sum_{n=0}^{\infty} \frac{a_{n}^{*}}{n !} x^{n}
$$

Theorem 41 of Hardy [8] asserts if $b_{n} \sim n^{\beta}, c_{n} \sim n^{\gamma}$, and

$$
\sum_{n=0}^{\infty} d_{n} x^{n}=\left(\sum_{n=0}^{\infty} b_{n} x^{n}\right)\left(\sum_{n=0}^{\infty} c_{n} x^{n}\right)
$$

then

$$
d_{n} \sim \frac{\Gamma(\beta+1) \Gamma(\gamma+1)}{\Gamma(\beta+\gamma)} n^{\beta+\gamma+1}
$$

Thus, by induction on $N$,

$$
\frac{a_{n}^{*}}{n !} \sim \frac{(A \Gamma(\alpha+1))^{N}}{\Gamma(N(\alpha+1))} n^{N(1+\alpha)-1} R^{-n}
$$

Now for $N$ as in the statement of the theorem, $N(1+\alpha)-1 \geqq 0$. By the remark at the beginning of Section $3 \mu(\varphi)$ exists for each monadic second order $\varphi$.

Notice that when $\alpha \geqq 0$ and $N=1$, and the theorem is equivalent to the remark at the beginning of Section 3.

Corollary 5. If $\mathscr{D}$ is the component $N$-coloring class, where $N \geqq 2$, for the class of unary functions or the class of partial unary functions then $\mu^{\mathscr{D}}(\varphi)$ exists for each monadic second order $\varphi$.

Thus, weighting unary functions to favor those with more components insures the existence of labeled asymptotic probabilities of monadic second order sentences.

\section{REFERENCES}

[1] Chang, C.C., Keisler, H.J.: Model theory. North-Holland, Amsterdam, 1973.

[2] Compton, K.J.: A logical approach to asymptotic combinatorics I: first order properties, Adv. Math. (to appear).

[3] Compton, K.J.: A logical approach to asymptotic combinatorics II: monadic second order properties, Adv. Math. (to appear).

[4] Compton, K.J.: The computational complexity of asymptotic problems II: permutations (in preparation). 
[5] Fagin, R.: Probabilities on finite models, J. Symb. Logic 41, 50-58 (1976).

[6] Glebskii, Yu.V., Kogan, D.I., Liogon'kii, M.I., Talanov, V.A.: Range and degree of realizability of formulas in the restricted predicate calculus. Kybernetik 5, 17-28 (1969). (Trans. (1972), 142-154).

[7] Goulden, I.P., Jackson, D.M.: Combinatorial enumeration. Wiley, 1983.

[8] Hardy, G.H.: Divergent series. Clarendon Press, Oxford, 1949.

[9] Kaufmann, M., Shelah, S.: On random models of finite power and monadic logic, Discrete Math. 54, 285-293 (1985).

[10] Lynch, J.F.: First-order probabilities or random unary functions. Trans. Am. Math. Soc. 287, 543-568 (1985).

[11] Rubel, L.: Maximal means and Tauberian theorems. Pacific J. Math. 10, 997-1007 (1960).

[12] Titchmarsh, E.C.: Theory of functions. Oxford University Press, London, 1952.

\section{Kevin J. Compton}

Department of Electrical Engineering

and Computer Science

University of Michigan

Ann Arbor

MI 48109-1109

USA 